\title{
CONTRIBUTIONS TO THE CRITICISM OF ZMARAGDUS'S EXPOSITIO LIBRI COMITIS.
}

The Work. The commentary which Zmaragdus, Abbot of St Michael's near Verdun, compiled on the Lectionary in the early years of the ninth century, was edited by Caspar Hedio ${ }^{1}$ and published by Georges Ulricher at Strasbourg in ${ }_{1536}$. The only other edition is the reprint of Hedio which Pitra superintended and issued in tom. cii of Migne's Patrologia Latina in 1851 . Pitra contented himself with the correction of some misprints and the addition of an appendix in which (pp. I I I I-I I 32) he called attention to some matters of interest connected with the compilation. From that time till 1905 , when Riggenbach used it as a source for Pelagius's commentary on the Epistles of St Paul, it appears to have attracted few readers. Yet it is far from being without interest and importance.

The word 'compiled' has been used advisedly of this commentary, for it is a compilation from earlier authors, and its value is that of its sources. There is almost nothing that is original in it ; but the author, obviously a man of great learning, has put it together, as he tells us in his preface, from the following authors:-Hilary, Jerome, Ambrose, Augustine, Cyprian, Cyril, Gregory, Victor, Fulgentius, John (i.e. Chrysostom ${ }^{2}$ ), Cassiodorus, Eucherius, Tyconius, Isidore, Figulus (?), Bede, Primasius, Pelagius, and Origen. He mentions that he abridged as well as transferred, but of the former process I have seen but little trace in confronting his work with its sources. These sources he was very careful to mark in the margins by using contractions of the names of the authors employed. This method had been practised by Bede, and our compiler may have borrowed the system from him.

The first editor had three MSS only at his disposal, of which one was both carelessly written and defective, while the others were complete, old and carefully written. By his own confession he went about his

\footnotetext{
1 Riggenbach, Unbeachtet gebliebene Fragmente des Pelagius-Kommentars su den Paulinischen Briefen (Beiträge zur Forderung christlicher Theologie, hrsg. von Schlatter u. Latgert $1 \times$ I) p. 7 .

2 The epithet is wanting in the MSS. For its history see Dom Baur's St Jean Chrysostome et ses Euvres (Louvain, 1907) p. $5^{8}$ ff.
} 
work in a wrongheaded way. He tells us he was 'adiutus maxime aùroypábors et ut sic dicam originalibus Catholicorum patrum'. In other words, where the MSS appeared to him wrong he looked out the passages in works of the Fathers themselves from which Zmaragdus was making the compilation. The text, as Zmaragdus wrote it, has thus been frequently obscured. Hedio also expanded the symbols in the margin indicating the sources, but he has frequently expanded them wrongly, has often omitted them, and has sometimes put them in at the wrong place. Pitra in his appendix has given a collation of the marginal symbols as they appear in the Boulogne MS, and has thus cleared away some of the errors. It is but fair to state that the MSS themselves are far from consistent in their reports of these symbols, and it is chiefly because I have had occasion to collate seven old MSS of Zmaragdus for the sake of his Pelagian quotations that I have taken the extra trouble of collating all the symbols in the margin.

The preparation of a new edition of Zmaragdus is a task which may be commended to any scholar of enthusiasm and industry. A moment's thought will shew that the MSS of Fathers used by him must have been at least as old as the end of the eighth century. His readings, therefore, are of importance to all editors of the authors above named. The future editor of Zmaragdus would also track all the quotations to their original sources, and note the exact references with or without mention of the differences in text between the original source and Zmaragdus's quotation from it. An index of sources would profitably close the volume. The task would be rather a long than a difficult one, as the MSS are fairly numerous and not far removed in time from the author. It is hoped that the present paper will smooth the future editor's path.

The Manuscripts. In this Journal (vol. vii p. 57I) I was able to enumerate twelve MSS. I have since then discovered three others: Zurich, Kantonsbibliothek (Rheinau) XII (saec. X), XXXII (saec. X), the latter being imperfect: Màdrid, Archivo Histórico Nacional I (saec. XII). I have further to point out that the Einsiedeln MS is imperfect; that the St Gall MS 435 is almost worthless; and that the Paris MS, as Dr Holder informs me, was formerly at Reichenau, and is, perhaps, the best of all. The Boulogne MS is probably a copy of the St Omer MS, or else both are copies of one original. The imperfect British Museum MS was one of those used by the first editor. ${ }^{1}$ It is probable that there are a good many other MSS of this work on the Continent. The difficulty of identifying them from catalogues is

1 The Commentary of Pelagius: the Problem of its Restoration (Proceedings of the British Academy vol. ii p. 43 I [ = p. 23]). 
very great, às they' are generally anonymous and may' be catalogued in so many different ways: From the early dates of the MSS it looks as if Zmaragdus's work had passed almost entirely out of use after the tenth century.

The Authorities Used. Hilary, Cyril; Fulgentius, Eucherius and Tyconius are very rarely cited, Cyprian perhaps never. The quotations' from Victor (of Capua probably), Pelagius, and Frigulus (?) have been discussed at some length by Pitra in his appendix, and those from Pelagius have been further discussed by Riggenbach and by Hellmann in his Sedulius Scottus. The identity of the mysterious Frigulus seems as far from being discovered as ever. In the preface he appears variously in MSS as Figulus, Frigulus, Fidolus, and the contractions in the body of the work which appear to represent him vary somewhat. The first editor has made one serious mistake. He has expanded $\overline{\mathbf{P}}$ always as 'Primasius', not having observed that $\overline{\mathrm{PR}}, \overrightarrow{\mathrm{PRI}}$, which occur only in comments on the Apocalypse, are the symbols for Primasius, and that $\overline{\mathrm{P}}$, which occurs only in comments on St Paul's epistles, means therefore Pelagius. It was, I believe, on this slender foundation, and on it only, that Gagney in the following year (1537) published under Primasius's name the anonymous commentary; which I have proved to be that of Cassiodorus, and which he had found in a MS now at Grenoble. ${ }^{1}$ All the quotations labelled $\bar{P}$ belong therefore either to the pure Pelagius commentary or to some adaptation of it. The quotations from $\overline{\mathrm{OR}}, \overline{\mathrm{ORI}}$, which the author gives in commenting on the Epistle to the Hebrews, are criticized by Riggenbach in his masterly monograph (Die altesten lateinischen Kommentare zum Hebraerbrief [Leipzig, 1907] p. 7 ff).

I have endeavoured to track all the quotations made on the Pauline Epistles to their original sources. The most remarkable thing about them is that neither the Ambrosiaster nor the Latin translation of Theodore of Mopsuestia was used by him. The following appear to be the only authorities he employed for this part of the work: Origen-Rufinus on Romans, Jerome on Galatians, Ephesians, Pelagius, Pseudo-Jerome on Second Corinthians, Chrysostom (Homilies on First and Second Corinthians?), Cassiodorus (Complexiones?), Isidore (On First Corinthians?), Cyril (on Philippians), Victor of Capua, Gregory, Chrysostom-Mucian on Hebrewes, Augustine De Diversis Quaestionibus, and the Pseudo-Augustinian Quaestiones Veteris et Novi Testamenti CXXVII.

\footnotetext{
1 Riggenbach agrees (Theol. Literaturblatt xxviii [1907] 74-75); Souter Comm. Pelag. ${ }^{28}$ [ $\left.=20\right]$.

3 The passages are given in the 'prolegomena! to my edition (CSEL. vol. 1 g08] p. xxvi).
} 
Errors of Printed Editions. In this section I propose to give all the certain corrections of wrong attributions in the editions, which collation of the MSS has enabled me to make. The MSS collated are the Bodleian, British Museum, Paris, Berlin, Einsiedeln, St Gall 424 and St Gall 435 ; but to save space and confusion I refrain from mentioning MSS, except where the truth is doubtful. ${ }^{2}$ The numbers and letters indicate pages of Migne P. L. cii. Though the fortunate possessor of a copy of the editio princeps, I have refrained from quoting it, because of its excessive rarity. The left-hand column contains the place of the error, the right-hand the correction.

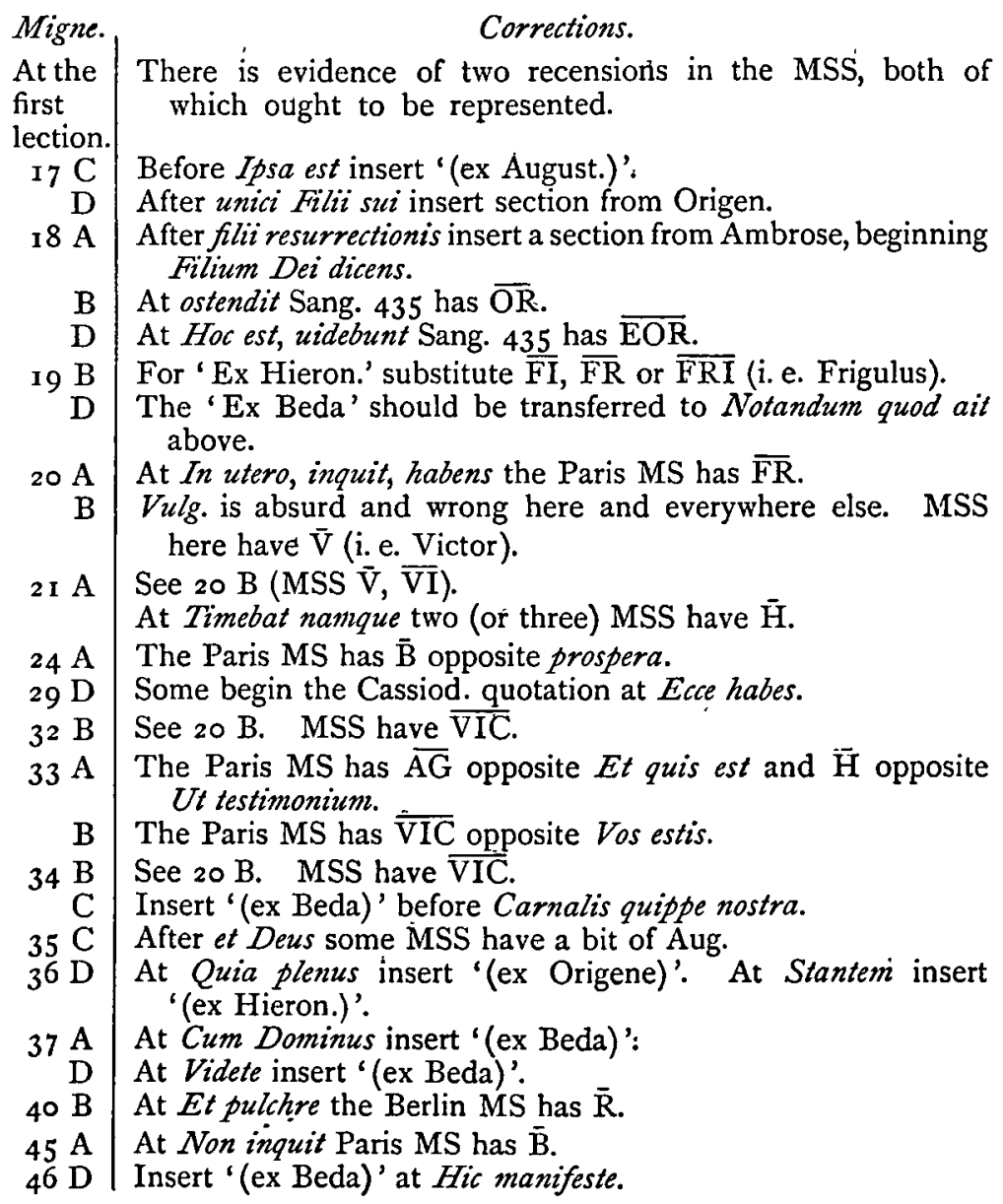

I I have refrained from using the readings of the Boulogne MS, recorded on pp. II15-1118, because $I$ have not verified them. 
Migne.

$47 \mathrm{D}$

C

C

\section{Corrections.}

At $I n$ hoc istarum Paris MS has $\overline{\mathrm{B}}$.

At Agnus insert '(ex Primas.)'.

At Magna vox insert '(ex Beda)'.

At Quid per insert '(ex Primas.)'.

At Concitharistae (cum citharistae codd.) Dei insert '(ex Beda)'. At Quid per canticum insert '(ex Primas.)'. At Hoc (hune codd.) enim canticum insert '(ex Cassiod.)'.

At Quod nulla insert '(ex Primas.)'. At Sedes Ecclesia insert '(ex Cassiod.)'. At Quattuor enim animalia insert '(ex Primas. )'.

At Singulariter canticum insert '(ex Beda)'.

At quando ueniat the Einsiedeln MS has $\overline{\mathrm{PRI}}$.

At redempti pretioso the Einsiedeln MS has $\overrightarrow{\mathrm{B}}$.

At Non dicit insert '(ex Frigulo)' (MSS have variously $\overline{\mathrm{F}}$, $\overparen{\mathrm{PRI}}, \widetilde{\mathrm{FL}}$.). Insert at Quia Babylon '(ex Io. Chrys.)'.

Insert at Possumus quoque '(ex Beda)'.

Insert at Aegyptus tenebrae '(ex Frigulo)' (MSS have $\overline{\mathrm{F}}, \overline{\mathrm{FI}}$ ). Insert at Quod dicit' 'ex Beda)'.

Insert at Hoc dicımus in '(ex Hieron.)'. Insert at Quod iterum '(ex Frigulo)' (MSS have $\overline{\mathrm{F}}$ ). Insert at Potest tamen '(ex Orig.)'.

Insert at In morte innocentium '(ex Beda)'.

Insert at Quod autem dicitur '(ex Hieron.)'.

Insert at Quid enim Rama '(ex Io. Chrys.)'. At Spiritaliter vox insert '(ex Beda)'.

At De Rachel insert '(ex Hieron.)'. At Figuraliter autem insert '(ex Beda)'.

At Plorat autem insert '(ex Hieron.)': At Spiritaliter Rachel insert '(ex Beda)'.

At Non utique perhaps insert '(ex August.)'. (MSS are confused here, giving $\overline{\mathrm{A}}, \overline{\mathrm{AG}}$ at Circumciditur which belongs to Ambrose, and at Non utique $\overline{\mathrm{A}}, \overline{\mathrm{AM}}, \overline{\mathrm{AMB}}$, though the passage does not appear in him.)

At Non solum $a b$ insert '(ex Ambros.)'.

At Secundum acceptam the Berlin MS gives $\overline{\mathrm{H}}$.

At Si enim insert '(ex [Pseudo-] August.)'.

Insert '(ex Pelag.)' at Mulier non (so the Berlin MS : the Bodleian and Paris MSS give $\overline{\mathrm{P}}$ not there, but at $I n$ eo autem, while the British Museum MS gives $\bar{A}$ at the latter place).

At Duae four MSS give $\overline{\mathrm{A}}$, which may mean Augustine. I cannot find the passage in Ambr.

66 A For '(Ex Hieron.)' read '(Ex Beda)'. At Nos quippe insert '(Ex Hieron.)'.

At Sed (et Migne) usque insert '(ex Beda)'.

.67 A (At Nam Anna MSS give $\bar{A}$ ).

C (At Multorum MSS give $\bar{A}$ ). 
Migne.

7 I

$72 \mathrm{~B}$

$73 \mathrm{D}$

$76 \mathrm{D}$

$77 \mathrm{~A}$

$80 \mathrm{C}$

D

8I B

$\mathrm{C}$

$82 \mathrm{~A}$

$\mathrm{B}$

$\mathrm{D}$

$8_{3} \mathrm{C}$

$84 \mathrm{~B}$

$85 \mathrm{C}$

$86 \mathrm{~A}$

$\mathrm{B}$

$87 \mathrm{~B}$

$89 \mathrm{C}$ 9I A

$104 \mathrm{D}$ $105 \mathrm{~B}$

$107 \mathrm{C}$ III B

$114 \mathrm{~B}$

$\mathrm{D}$

$115 \mathrm{~B}$

Corrections.

At Pulchre autem insert '(ex Hieron.)'.

See 20 B. MSS give $\overline{\mathrm{V}}$. At Alii dicunt insert '(ex Frigulo)' (MSS give $\overrightarrow{\mathrm{F}}$ ).

At Natiuitate Einsiedeln MS gives $\bar{A}(?)$.

Some MSS omit Dum enim-discutimus, aberremus.

At Donum substitute '(ex Pelag.)' for '(ex Primas.)'.

At De ministerio substitute '(ex Pelag.)' for '(ex Primas.)'. At Maxime substitute '(ex Pelag.)' for '(ex Primas.)'.

At Tota puritas insert '(ex Pelag.)'.

At $A c$ si insert '(ex Pelag.)'.

At Hoc si substitute '(ex Pelag.)' for '(ex Primas.)'.

At $N e$ per substitute '(ex Pelag.)' for '(ex Primas.)'.

At Propter gaudium substitute '(ex Pelag.)' for '(ex Primas.)'.

At Habenda the Einsiedeln MS gives $\bar{H}$.

At Superbe (Superbia ed.) substitute '(ex Pelag.)' for '(ex Primas.)'.

At Quod Dominus Paris MS has $\overline{\mathrm{I}}$.

The Bodleian and Paris MSS. give the $\overline{\mathrm{AG}}$ of $85 \mathrm{D}$ at Hora ergo and Nondum venit respectively.

The Bodleian MS gives $\overline{\mathrm{B}}$ at Sciebat.

The Bodleian MS gives $\overline{\mathrm{B}}$ at Et quidem potuit.

The Einsiedeln MS gives $\overline{\mathrm{P}}$ at Modo meruisti.

At Qui sibi insert '(ex Orig.)'.

At Nolite in substitute '(ex Pelag.)' for '(ex Primas.)'. At Si malum insert '(ex Orig.)'.

At Humana substitute '(ex Pelag.)' for '(ex Primas.)'.

At Quod uestrum substitute '(ex Pelag.)' for '(ex Primas.)'.

At Aut fugiendo substitute '(ex Pelag.)' for '(ex Primas.)'.

Insert '(ex Pelag.)' at Ylle dicitur.

The Bodleian and Paris MSS give another $\overline{\mathrm{B}}$ at In testimonium illis.

At Suscitant the Einsiedeln MS gives $\overline{O R}$.

For '(Ex Hieron.)' substitute '(ex Frigulo)'. (MSS give $\overline{\mathrm{FR}}, \overline{\mathrm{F}}$.)

At Ad undecimam the Einsiedeln MS gives A. At Pensate, fratres the Bodleian MS gives $\overline{\mathrm{G}}$.

At Id est the Berlin MS gives $\overline{\mathrm{B}}$.

At Hoc est, si the British Museum MS gives $\overrightarrow{\mathrm{OR}}$.

At Ut minus the Berlin MS has $I \bar{H}$.

The section from Bede is sometimes not at this point but at the end of the passages for this day.

The Einsiedeln MS omits Numquid iam ... campum quaesiuit.

Transfer the '(Ex Greg.)' from Et tamen to Mirum quomodo above.

For (Vulg.) see z० B. MSS have $\overline{\mathrm{VI}}, \overline{\mathrm{VIC}}, \overline{\mathrm{VT}}$.

At Leuem MS Sang. 424 has $\overline{\mathrm{P}}$, and at Non excidit $\overline{\mathrm{A}}$.

At Exemplo substitute. '(ex Pelag.)' for '(ex Primas.)'. 
Migne.

II 5 B

$\mathrm{C}$

$117 \mathrm{D}$

I 19 B

$\mathrm{C}$

C, D

$120 \mathrm{~B}$

$\mathrm{D}$

I 2 I B

$\mathrm{D}$

$125 \mathrm{~B}$

$126 \mathrm{D}$

$127 \mathrm{~B}$

$128 \mathrm{C}$

$129 \mathrm{C}$

I $30 \mathrm{~A}$

I3 $\mathrm{C}$

D

$133 \mathrm{~A}$

I $36 \mathrm{C}$

$137 \mathrm{C}$

$\mathrm{D}$

$\times 38 \mathrm{~B}$

$139 \mathrm{C}$

$142 \mathrm{C}$

I $48 \mathrm{D}$

$149 \mathrm{~A}$

D

$15 \circ \mathrm{B}$

C

$160 \mathrm{C}$ $166 \mathrm{~A}$

$168 \mathrm{C}$

$174 \mathrm{D}$

I75 A

\section{Corrections.}

At Sic ergo substitute '(ex Aug.)' for '(ex Ambrosio)'. At Ostendit substitute '(ex Pelag.)' for '(ex Primas.)'. At Aenigma est substitute '(ex Aug.)' for '(ex Amb.)'. At Quid isti designant the British Museum MS has $\overline{\mathrm{G}}$, suggesting that some other author has been used just before. See $9 \mathrm{r} C$.

See 9 I C.

There is some uncertainty about the source and the labelling of the section Id est... institutum. The Berlin MS gives $\overline{\mathrm{P}}$, and the British Museum MS gives $\overline{\mathrm{P}}$ at Nemo instead of $\overline{\mathrm{H}}$. For '(ex Ambros.)' substitute '(ex [Pseudo-] Aug.)'. For '(ex Ambr.)' substitute '(ex Aug.)'. See 9 I $C$. Substitute '(ex Aug.)' for '(ex Ambr.)'. Substitute '(ex Aug.)' for '(ex Ambros.)'. (The Berlin MS has $\overline{\mathrm{P}}$ at Tentatur quadraginta !)

See $20 B$. MSS have $\overline{\mathrm{V}}, \overline{\mathrm{VI}}$.

Substitute '(ex Aug.)' for '(ex Amb.)', and for '(Ex Fulg.)' '(ex Frigulo)': in the latter case MSS have $\overline{\mathrm{FRI}}, \overline{\mathrm{F}}$.

At Dicens diabolus insert '(ex Beda)'.

See 9 I C.

At Uas, id est insert '(ex Pelag.)'.

The Bodleian MS gives $\overline{\mathrm{B}}$ opposite Et alias oues.

The Bodleian MS gives $\overline{\mathrm{B}}$ opposite Notandum sane.

See 9 r C.

The Bodleian and St Gall 435 MSS give $\overline{\mathrm{B}}$ at Non haec.

(The Paris MS gives $\overline{\mathrm{R}}$ opposite Iste est digitus.)

At Igitur manus the Bodlelan and Paris MSS give $\bar{A}$.

For '(Ex Beda)' substitute '(ex Aug.)',

At Inmundus insert '(ex Hieron.)'.

At Tesus ergo Einsiedeln MS gives $\bar{G}(?)$.

See 9 r C.

The MSS give $\bar{A}$. Probably substitute 'Aug.' for 'Ambr.'.

The Paris MS gives $\overline{\mathrm{P}}$ at Id est Ecclesiae. This is right : therefore expand to '(ex Pelag.)':

At Qui possunt MSS give $\bar{A}$ : therefore expand to either '(ex Aug.)' or '(ex Ambr.)'.

At Nunc igitur insert '(ex Isidoro)'.

At Genus humanum insert '(ex August.)'.

Transfer '(ex Orig.)' from Si enim to et in hac ipsa, and see Riggenbach, op. cit. p. $7 \mathrm{ff}$.

Insert '(ex August.)' at Quem uult.

At Post duos dies insert '(ex Hieron.)'. At Hoc sane iuxia insert '(ex Beda)'.

B At Mulier ista. Maria insert '(ex Beda)'. 
Migne.

I $76 \mathrm{D}$

$177 \mathrm{C}$

I $78 \mathrm{~A}$

C

$179 \mathrm{~B}$

C

I $80 \mathrm{~A}$

B

I $8 \mathrm{I} \mathrm{A}$

$B$

$182 \mathrm{~A}$

$B$

C

$183 \mathrm{~B}$

D

$184 \mathrm{~A}$

C At Ut compleretur insert '(ex Hieron.)'. At Uelauerunt insert '(ex Beda)'.

D At Non quod alterius insert '(ex Hieron.)'.

$185 \mathrm{D}$ At Hoc est quod insert '(ex Beda)'.

I86 C At Pilatus autem accepit insert '(ex Hieron.)':

D At Pro regia insert '(ex Beda)'.

I87 C At In chlamide coccinea insert '(ex Hieron.)'.

189 C Opposite Postquam autem Bodleian MS has $\overline{\mathrm{N}}$, perhaps by error for $\overline{\mathrm{H}}$, as elsewhere.

D My MSS have no '(Ex August.)'.

' $90 \mathrm{D}$ At Blasphemabant insert '(ex Hieron.)'.

19r B The Bodleian and Paris MSS have $\overline{\mathrm{G}}$ opposite Nunc autem manet, and $\overline{\mathrm{R}}$ the former opposite Fidem namque, the latter opposite Spem habuit.

D At uelut impium scelus insert '(ex Victore)': MSS have $\overline{\mathrm{VI}}, \overline{\mathrm{V}}$.

r 92 A At Rationis igitur insert ' (ex Hieron.)'.

I93 A My MSS have no '(ex Beda)', but opposite Lignum aduersus insert '(ex Gregor.)'.

B Insert at Velum templi scissum '(ex Hieron.)' and withdraw it from In Euangelio.

$\mathrm{C}$ At Quaerendum est utrum Bodleian MS has $\overline{\mathrm{B}}$.

$194 \mathrm{C}$ See 20 B.' MSS have $\overline{\mathrm{VI}}, \overline{\mathrm{V}}$.

196 A At Decurio uocatur insert '(ex Beda)'. At Magnae quidem Ioseph insert '(ex Hieron.)'.

D After promisist $i$ the Einsiedeln and Berlin MSS add a little: At De monumento insert '(ex Beda)'.

$198 \mathrm{D} \mid$ At $N$ Non suffecerat insert '(ex Hieron.)'. 
Migne.

$200 \mathrm{~A}$

B

C Substitute '(ex Cyrill.)' for '(Ex Chrys.)'. MSS have again $\overline{\mathrm{CY}}$.

D At Nomen habitus dicitur insert '(ex August.)'.

$201 \mathrm{D}$ At Quomodo accepit insert '(ex Victore)". MSS have $\overline{\mathrm{VI}}, \overline{\mathrm{V}}$, $\overline{\mathrm{VIG}}($ sic).

$202 \mathrm{~A}$

At Praeter Einsiedeln MS has $\overline{A C M}$ (i. e. it corrects an orig. Aug. to Ambr.).

B After this day follows the Passio in the Paris and St Gall 424 MSS.

$220 \mathrm{~B}$ At Haec MS Sang. 424 has $\dot{\mathrm{V}}$.

$222 \mathrm{C}$ For '(Ex August.)' substitute '(Ex Frigulo)'. MSS have $\overline{\mathrm{FRI}}, \overline{\mathrm{FE}}, \overline{\mathrm{FV}}$.

223 B Some give '(ex August.)' at In prima, etc.

C See 20 B. MSS have $\overline{\mathrm{VI}}, \overline{\mathrm{V}}$.

225 A At In Evangelio Lucae insert '(ex Beda)', but British' Museum MS has $\overline{\mathrm{G}}$.

$226 \mathrm{~A}$ At Et introeuntes Bodleian MS has $\mathrm{F}$.

B At Notandum uero insert '(ex Gregor.)', but the Einsiedeln MS gives $\bar{G}$ at scribit enim, the Paris MS at Illae ergo mulieres.

229 A Insert '(ex Beda)' at Id est, Pater.

Insert '(ex Ambros. [August. ?])' at Christus passus est (MSS give $\bar{A}$, except Einsiedeln MS which gives $\overline{A M}$ ).

B Insert '(ex August.)' at Quis suscitauit.

233 A At Nihil simplex insert '(ex Gregor.)' (but Bodleian MS gives $\bar{A}$ ).

D At Iam erat insert '(ex August.)'.

234 D At Omnes quibus insert '(ex August.)'.

$235 \mathrm{~A}$

$236 \mathrm{~A}$

$237 \mathrm{~A}$

B

$\mathrm{D}$

$238 \mathrm{~B}$

$240 \mathrm{~B}$

$24 \mathrm{IB}$

$245 \mathrm{~A}$

$246 \mathrm{~A}$

$25 \mathrm{ID}$

$252 \mathrm{~A}$

$253 \mathrm{~B}$

At Aliter namque insert ' (ex Beda)'.

My MSS have no '(ex Beda)'.

At Notandum quod Sang. 435 has $\overline{\mathrm{B}}$.

My MSS have no '(ex Greg.)'.

My MSS have no '(ex August.)'.

At In corde the Berlin MS has B (erased).

At $N o n$ solum insert '(ex Beda)'.

Substitute '(ex Beda)' for '(ex Primas.)'. (The editor mistook

a $\bar{B}$ for a $\bar{P}$, and then expanded wrongly, as always.)

$257 \mathrm{~B}$ At Pensandum the Berlin MS gives $\overline{\mathrm{G}}$ (rightly?). 
Migne.

$263 \mathrm{D}$

$264 \mathrm{~B}$

$(274 \mathrm{C}$

(280 D $282 \mathrm{~B}$ $285 \mathrm{D}$

$295 \mathrm{~B}$ $300 \mathrm{~A}$

$(3 \circ 3 \mathrm{~B}$ $306 \mathrm{~A}$

3 I $\mathrm{D}$

3 I $2 \mathrm{D}$

(3I6 D

$328 \mathrm{~B}$

$332 \mathrm{~A}$

$339 \mathrm{C}$

343 D

349 A

$35^{\circ} \mathrm{C}$

$355 \mathrm{D}$

$35^{8} \mathrm{C}$ $360 \mathrm{C}$

$363 \mathrm{~A}$ $367 \mathrm{~A}$ $369 \mathrm{~B}$

$374 \mathrm{~B}$

$375 \mathrm{~A}$

$377 \mathrm{~B}$

$378 \mathrm{~A}$

$379 \mathrm{D}$ $3^{80 \mathrm{~A}}$

$\mathrm{C}$ $\left(3^{8} \times \mathrm{A}\right.$

\section{Corrections.}

Insert '(ex August.)' somewhere here. One MS gives it at Sed ut legi, another at De Christo, another at In quo subauditur.

At Id est quattuor insert '(ex Frigulo)': Berlin MS has $\overline{\mathrm{FRI}}$.

At Una sabbati the British Museum MS gives $\overline{\mathrm{G}}(?)$ and the Berlin MS gives $\overline{\mathrm{B}}$.)

At $E t$ hoc Berlin MS gives $\overline{\mathrm{AG}}$.)

At Quod autem dicit insert '(ex Beda)'.

Before Ostensa some MSS (for example, the Einsiedeln) give a passage beginning with Fecit.

At Velocitas the Einsiedeln MS gives $\overline{\mathrm{H}}$.

At Sic et insert ' (ex Beda)'.

Here British Museum MS ends.)

At Rogatus insert '(ex Beda)'.

At Cum autem dicatur the Einsiedeln MS has $\bar{A}$.

At. Quid in his Einsiedeln MS has $\overline{\mathrm{G}}($ ?).

The Einsiedeln MS ends here.)

At Probatio the Bodleian MS has $\overline{\mathrm{G}}$.

My MSS are without '(ex Beda)'.

At Princeps scilicet the Paris MS has $\overline{\mathrm{B}}$.

At Quomodo the Berlin MS has $\overline{\mathrm{B}}$.

At Nonnulli autem insert '(ex Gregor.)'.

At $O$ quanta insert ' (ex Gregor.)'.

For '(ex Hieron.)' substitute '(ex Gregor.)'.

At Hanc ergo insert '(ex Beda)'.

At Quia non solum insert '(ex Beda)'.

At ad conuescendum Bodleian MS has $\overline{\mathrm{G}}$.

At Si quis insert '(ex Orig.)'.

At Quidam etiam the Paris and Berlin MSS give $\bar{P}$.

At Dimittere nos insert '(ex Victore)' (MSS have $\overline{\mathrm{VIC}}, \overline{\mathrm{V}}$ ).

At Dominum autem Berlin MS gives $\overline{\mathrm{S}}$.

My MSS have no '(ex Beda)'.

(At Potest the Berlin MS has $\bar{q}$ ).

At Non quod insert '(ex Hieron.)'.

At Plerumque iustitia insert '(ex Beda)'.

At Qui enim the Berlin MS gives $\bar{M}$.

At Non inmerito insert '(ex Ambr.)'.

At Sicera the Berlin MS has $\bar{M}$ : possibly therefore the $\bar{M}$ at 380 A should be $\bar{B}$.)

My MSS are without the '(ex Amb.)'.

My MSS are without the '(ex Hier.)', but the Bodleian MS has $\overline{\mathrm{SQR}}$ at Hoc est ab $(382 \mathrm{~B})$.

At Neque poterat the Paris MS has $\bar{M}$.

See 20 B. MSS have $\overline{\mathrm{VI}}, \overline{\mathrm{V}}$.

For '(ex Iosepho)' the Paris MS has $\overline{\mathrm{B}}$, no doubt rightly.

At Philippus iste put the '(Ex Hieron.)' which is given below, and erase it there.

VOL. IX. 


\section{THE JOURNAL OF THEOLOGICAL STUDIES}

\begin{tabular}{|c|c|}
\hline e. & Corrections. \\
\hline $\mathrm{B}$ & 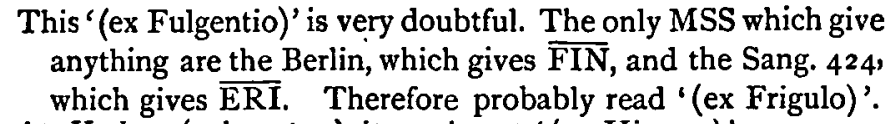 \\
\hline & At Verbum (v. l. uotum) ip \\
\hline & $\begin{array}{l}\text { At Hic discimus MSS have } \overline{\mathrm{A}} \text { : therefore perhaps expand '(ex } \\
\text { Ambr.)'. }\end{array}$ \\
\hline & $\begin{array}{l}\text { My MSS are without '(ex Orig.)'. } \\
\text { See gI C. }\end{array}$ \\
\hline & See 9r C. \\
\hline & $\begin{array}{l}\text { At Id est insert ' (ex Frigulo)'. (The Paris MS has } \overline{\mathrm{FRI}} \text {, the } \\
\text { Sang. } 424 \text { has } \overline{\mathrm{AG}} \text { by anticipation.) }\end{array}$ \\
\hline & $\begin{array}{l}\text { At Gehenna nomen insert '(ex Frigulo)'. (MSS have } \overline{\mathrm{FRI}} \text {, } \\
\overline{\mathrm{FRA}}, \overline{\mathrm{FI}} \text {.) }\end{array}$ \\
\hline B & $\begin{array}{l}\text { At Docet utique insert ' (ex Hilar.)'. (MSS have } \overline{\mathrm{HIL}}, \overline{\mathrm{NIL}} \text {.) } \\
\text { At Si in mentem perhaps we ought to substitute '(ex Ambr.)'. } \\
\text { (MSS have } \bar{A} . \text {.) }\end{array}$ \\
\hline & See 9 \\
\hline & See \\
\hline & $\begin{array}{l}\text { At Romanis militibus the Bodleian MS has } \overline{\mathrm{NIL}} \text { : therefore } \\
\text { insert '(ex Hilario)'. }\end{array}$ \\
\hline C & $\begin{array}{l}\text { At Ergo stipendium the Bodleia } \\
\text { Insert '(ex Pelag.)' at Non dix }\end{array}$ \\
\hline & $\begin{array}{l}\text { For '(ex Beda)' substitute '(ex August.)' [or '(ex Ambr.)']. } \\
\quad \text { (MSS have } \bar{A} \text {.) }\end{array}$ \\
\hline & See 9 I C. \\
\hline . & $\begin{array}{l}\text { My MSS are defective in omitting the '(ex Pelag.)', which } \\
\text { should come at Hoc enim dicit, the '(Ex Orig.)' at } \\
\text { Qui ergo, and the '(ex Pelag.)' which should come at } \\
\text { 412 A. }\end{array}$ \\
\hline & $\begin{array}{l}\text { See } 9 \\
\text { MSS }\end{array}$ \\
\hline & At $S$ \\
\hline & The e \\
\hline & $\begin{array}{l}\text { The last two-thirds of the Epistle for 'Hebdomada X post } \\
\text { Pentecost.' differ greatly in MSS. }\end{array}$ \\
\hline & $\begin{array}{l}\text { After tribulationibus probat there is in some MSS an extract } \\
\text { labelled } \bar{A} \text {. In some of these MSS the rest of the comm. } \\
\text { on the Lesson is cited only from } \overline{\mathrm{IS}} \text { and } \overline{\mathrm{A}} \text {. The Isidore } \\
\text { extract begins Mortuo Aaron. }\end{array}$ \\
\hline $\mathrm{C}$ & $\begin{array}{l}\text { See 91 C. Substitute '(ex August.)' for '(ex Hieron.)'. } \\
\text { (MSS have } \bar{A} \text {.) }\end{array}$ \\
\hline & See gr C. \\
\hline & rightly without ('Ex \\
\hline & Opposite Ablata quippe the Paris MS \\
\hline
\end{tabular}


Migne. Corrections.

418 C Opposite Cadus Graece the Paris MS has $\overrightarrow{\mathrm{G}}$.

420 A My MSS are without '(ex Orig.)'.

42 I D Substitute '(ex Gregor.)' for '(ex Beda)'.

427 D At At cum ea the MS Sang. 424 has A.

$433 \mathrm{D}$ See $91 \mathrm{C}$.

437 A See 9 r C.

$439 \mathrm{D}$ See 9 I C.

$443 \mathrm{~A}$

At Hic certe insert '(ex August.)'.

B See 91 C.

( $\mathrm{C}$ At $A c$ si the Paris MS has $\overline{\mathrm{A}}$ wrongly, and the Berlin MS has a letter erased.)

D Opposite Ita timor the Berlin MS has a letter erased.

444 A See 9r C. Substitute '(ex Pelag.)' for the second '(ex

Primas.)' also, and transfer it to Quidam mediatorem Mosen.

$445 \mathrm{~A}$

$446 \mathrm{~B}$

C Opposite Dum legisperito the Berlin MS has one letter erased.

D Opposite Homo the Berlin MS has one letter erased.

447 D Opposite Iumentum eius Paris MS has NIL (IL in ligațre): this points to HIL.

449 B At Hoc tripliciter insert '(ex Hieron.)'.

45 I C The words de Neotericis Graecum uersum transferens, omitted

in at least two MSS, would appear to be no part of the text.

$454 \mathrm{C}$ At Samaritanus Sang. 435 gives $\overline{\mathrm{H}}$.

$\mathrm{D}$ At Unum si Sang. 435 gives $\overline{\mathrm{S}}$.

456 A See 91 C.

457 A

B See 9 r C.

$45^{8} \mathrm{~B}$ See $9 \mathrm{I} \mathrm{C.} \mathrm{At} I d$ est, qui insert '(ex Hieron.)', and remove it from before Praeuidens.

C See 9 I C. Correct '(Ex Primas.)' (alt.) to '(ex Hieron.)'. $459 \mathrm{~A}$

B

$460 \mathrm{~B}$

$46 \mathrm{r} \mathrm{A}$

$463 \mathrm{~B}$

$464 \mathrm{D}$

$465 \mathrm{~A}$

B

$467 \mathrm{D}$

$473 \mathrm{~B}$

See $9 \mathrm{r}$ C.

See $9 \mathrm{r}$ C.

At id est insert.'(ex Pelag.)'.

At Patietur insert '(ex August.)'.

'(Ex Fulg.)' is probably wrong. Substitute '(Ex Frigulo)':

MSS have $\bar{F}, \overline{F I}$. Substitute for '(Ex Hilar.) ' '(ex Hieron.)'.

At Haec est communio insert '(ex August.)' [or '(ex Ambr.)'].

Insert at Naim ciuitas '(ex Beda)'.

At Ecce defunctus, at Qui bene and at Nam et electus the Berlin MS has a letter erased in margin.

At Et haec uidua the Berlin MS has a letter erased in margin. My MSS have nothing here.

For '(Ex Fulg.)' substitute '(ex Frigulo)'. (MSS have FRI, $\overline{\mathrm{FI}}, \overline{\mathrm{F}}$.$) At Pharisaei et insert '(ex Hieron.)'.$ 
Migne.

$474 \mathrm{C}$

$478 \mathrm{C}$

483 B

486

$492 \mathrm{~A}$

B

D

$493 \mathrm{~A}$

D

$494 \mathrm{~A}$

D

$500 \mathrm{D}$

$502 \mathrm{~A}$

$506 \mathrm{D}$

$508 \mathrm{D}$

$5 \mathrm{roD}$

5 II A

C

$517 \mathrm{~B}$

C

$518 \mathrm{~B}$

$521 \mathrm{C}$

$523 \mathrm{D}$

$524 \mathrm{~B}$

$525 \mathrm{D}$

$526 \mathrm{~A}$

$528 \mathrm{~A}$

C

$532 \mathrm{C}$ $53^{8} \mathrm{C}$ 545 B

$546 \mathrm{D}$

547 B

548 B

Corrections.

Opposite in reliquis Paris $\mathrm{MS}$ has $\overline{\mathrm{B}}$.

Two MSS give the $\overrightarrow{\mathrm{B}}$ at Nota quod, not at Notandum.

At Curatio paralytici insert '(ex Beda)'.

My MSS give none of the ascriptions.

At Aestimo insert '(ex August.)'.

Opposite sed a quibusdam the Berlin MS gives $\overline{\mathrm{H}}$.

At Non aduersus the Berlin MS gives $\overline{\mathrm{P}}$, probably by anticipation : the others give nothing.

See 9r C. For '(Ex August.)' substitute '(Ex Hieron.)'.

See $91 \mathrm{C}$.

See 9 I C.

See 9r C. At Fidelis quasi insert '(ex Hieron.)'.

My MSS have no '(Ex August.)'.

The '(Ex August.)' should be transferred to Id est, me, where the Bodleian and Berlin MSS give it, but the ascription to Aug. is an error, probably going back to the archetype : read '(ex Pelag.)'.

At Octauum signum insert '(ex Hieron.)'.

Substitute '(Ex Beda)' for '(Ex Primas.)'. Cf. 253 B.

See $20 \mathrm{~B}$ (Paris MS has $\overline{\mathrm{VI}}$ ). At Ubi tetragrammaton MS Sang. 424 has $\overrightarrow{O R}$.

For '(Ex Eucherio)' substitute '(Ex Frigulo)'. (MSS have $\overline{\mathrm{FRI}}, \overline{\mathbf{F}}$.)

For '(Ex Fulg.)' substitute '(ex Frigulo)'. (MSS have $\overline{\mathrm{FI}}, \overline{\mathrm{F}}$.)

For '(Ex Fulgentio)' substitute '(ex Frigulo)'. (MSS have $\overline{\mathrm{ER}}, \overline{\mathrm{F}}$.)

See 9I C, but the '(Ex Pelag.)' ought to come at Sicut audire.

At Quoniam Iesse insert '(ex Orig.)'.

At Quod uero dicit insert '(ex Beda)'.

Substitute '(Ex Frigulo)' for '(Ex Fulgent.)'. (MSS have $\overline{\mathrm{FRI}}, \overline{\mathrm{FR}}$.)

At His uerbis insert '(ex Gregor.)'.

At Quod ergo insert '(ex Gregor.)'.

At Soluere calciamentum insert '(ex Victore)'. (MSS have $\overline{\mathrm{VI}}$.)

At Bethania villa insert '(ex Hieron.)'.

At Permisit tradi insert '(ex Pelag.)'. (MSS have $\overline{\mathbf{P}}$.)

At Numquid accusare MS Sang. 424 wrongly gives $\overline{\mathrm{OR}}$.

For '(Ex Beda)' the Paris MS gives $\overline{\mathrm{G}}$.

At Aliter lumbos insert '(ex Gregor.)'.

For '(Ex Fulg.)' the Paris MS,reads N : read probably '(Ex Frigulo)'.

For '(Ex Hieron.)' substitute '(ex Frigulo)'. (Berlin MS has $\overline{\mathbf{F}}$.) At Crebris parabolarum insert '(ex Hieron.)', removing it from Thesaurus iste.

Opposite cum autem venerit Paris MS has $\overline{\mathrm{N}}$, but whole section is Jerome. 


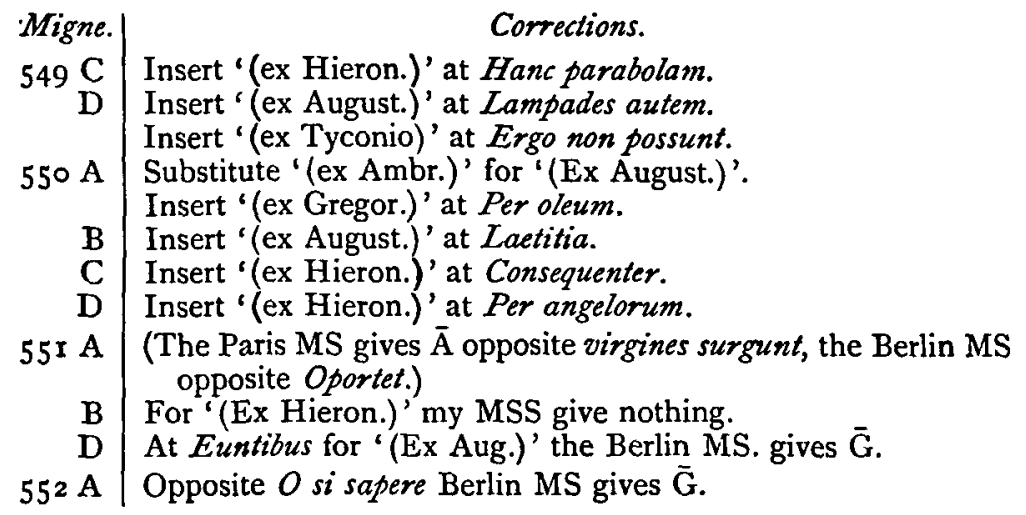

The defects of the printed editions in this matter of citation are sufficiently apparent. They can be paralleled by defects in the texts presented. The student is warned not to trust the editions for critical work of any sort. It is hoped that the present paper will save a good deal of vain searching after passages wrongly ascribed. I have left the MSS to speak for themselves, and have rarely searched in the original authors for verification of their testimony, except in the case of comments on the Pauline Epistles.

A. Souter.

\section{RENDERINGS OF THE INFINITIVE ABSOLUTE IN THE LXX.}

A very common usage of the Hebrew language is that of the infin. abs. of a verb in conjunction with the finite parts of the same verb, to

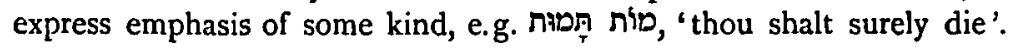
The translators of our English A.V. have shewn much skill and versatility in their renderings of this form of expression Most often they employ an adverb or an adverbial phrase. The following are a few examples :-Gen. ii 16 'Thou mayest freely eat', xvii I $3_{3}$ 'must needs be circumcised', xxxi 30 'sore longedst', $x{ }^{1} 15$ 'indeed I was stolen away', I Sam. ii 27 'plainly appear', vi 3 'in any wise return', Is. xxiv 19 ' The earth is utterly broken down, the earth is clean dissolved, the earth is moved exceedingly'.

The Greek translators have, for the most part, employed one of two methods for rendering the infinitive absolute, one of which is not 